\title{
Dry-Transfer of Aligned Multiwalled Carbon Nanotubes for Flexible Transparent Thin Films
}

\author{
Matthew Cole, ${ }^{1}$ Pritesh Hiralal, ${ }^{1,2}$ Kai Ying, ${ }^{1}$ Chi Li, ${ }^{1,3}$ Yan Zhang, ${ }^{1}$ Kenneth Teo, ${ }^{4}$ \\ Andrea Ferrari, ${ }^{1}$ and William Milne ${ }^{1,5}$ \\ ${ }^{1}$ Electrical Engineering Division, Department of Engineering, University of Cambridge, $9 \mathrm{JJ}$ Thomson Avenue, \\ Cambridge CB3 OFA, UK \\ ${ }^{2}$ Nokia Research Centre, Broers Building, 21 JJ Thomson Avenue, Cambridge CB3 OFA, UK \\ ${ }^{3}$ Display Research Centre, School of Electronic Science and Engineering, Southeast University, Nanjing 210096, China \\ ${ }^{4}$ AIXTRON Ltd., Anderson Road, Swavesey CB24 4FQ, UK \\ ${ }^{5}$ Department of Information Display, Kyung Hee University, Seoul 130-701, Republic of Korea
}

Correspondence should be addressed to Matthew Cole, mtc35@cam.ac.uk

Received 14 October 2011; Accepted 26 December 2011

Academic Editor: Teng Li

Copyright (C) 2012 Matthew Cole et al. This is an open access article distributed under the Creative Commons Attribution License, which permits unrestricted use, distribution, and reproduction in any medium, provided the original work is properly cited.

\begin{abstract}
Herein we present an inexpensive facile wet-chemistry-free approach to the transfer of chemical vapour-deposited multiwalled carbon nanotubes to flexible transparent polymer substrates in a single-step process. By controlling the nanotube length, we demonstrate accurate control over the electrical conductivity and optical transparency of the transferred thin films. Uniaxial strains of up to $140 \%$ induced only minor reductions in sample conductivity, opening up a number of applications in stretchable electronics. Nanotube alignment offers enhanced functionality for applications such as polarisation selective electrodes and flexible supercapacitor substrates. A capacitance of $17 \mathrm{~F} / \mathrm{g}$ was determined for supercapacitors fabricated from the reported dry-transferred MWCNTs with the corresponding cyclic voltagrams showing a clear dependence on nanotube length.
\end{abstract}

\section{Introduction}

Carbon nanotubes, one-dimensional high aspect ratio carbon allotropes, have received significant interest in the past two decades as a viable candidate material to replace the industry pervading, and increasingly expensive, transparent conductor, indium tin oxide (ITO). Although highly transparent and highly conductive, ITO exhibits poor flexing performance due to microcrack formation. Carbon nanotube networks are extremely flexible [1-3], highly conductive [46] and offer impressive optical transparency [4, 7-9]. Nevertheless, in order to fully exploit these features, films must be free-standing or supported on substrates of similar opacity and flexibility, namely, polymers and elastomers. Little work on the direct deposition of aligned nanotubes onto polymers has been reported [10], and as a result few applications exploit the properties of pristine nanotubes. Direct nanotube deposition on polymers necessitates substantial reductions in growth temperatures. This most often results in the deposition of inflexible pyramidal carbon nanofibres [11] rather than highly graphitic and conductive nanotubes.

A variety of solution processes have been explored to produce nanotube thin films [5, 12-14]. Nevertheless, such detrimental chemi douche processing, employing aggressive sonication and costly acid treatments, ultimately degrades the nanotubes electronic properties. Nanotube alignment has been demonstrated in a number of ways [15-17] though solution processing produces isotropic networks lacking the advantageous structural anisotropy associated with the initial as-grown one-dimensional nanostructures. Consequently, a wet-chemistry-free, inexpensive, and facile transfer technique, that retains the nanotubes alignment, is necessary for applications such as flexible optoelectronic polarisers and supercapacitors for next-generation energy storage [18-21] and display technologies.

Carbon nanotube-based thin films offer extremely high surface areas which make them functionally desirable for high-density energy storage, though binderless processes 


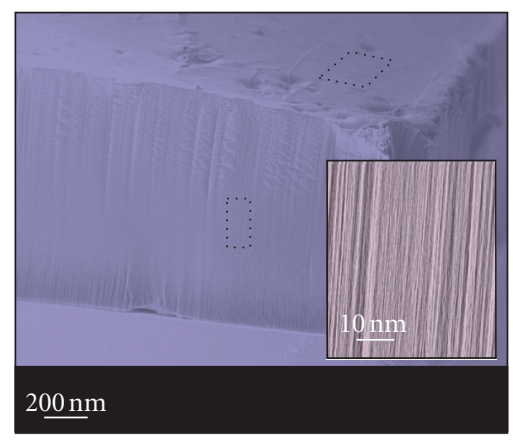

(a)



(b)

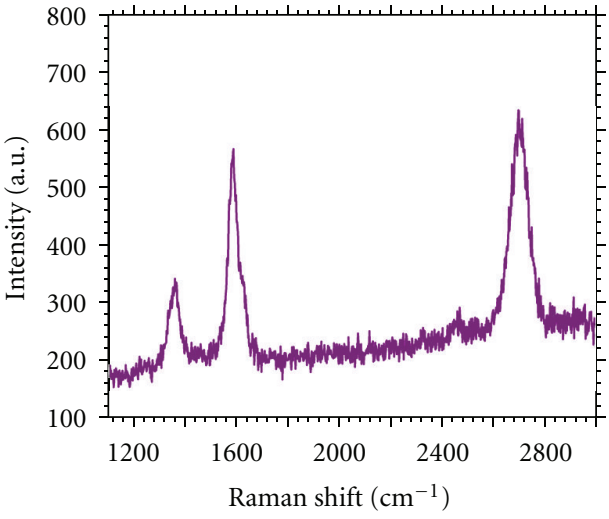

(c)

FIGURE 1: (a) Side profile SEM micrograph of the source MWCNTs. Inset: high-resolution SEM micrograph of the lower highlighted area demonstrating the extremely linear growth. (b) Areal SEM micrograph of the upper highlighted area in (a) illustrating the spaghetti-like morphology of the forest surface. Inset: HR-TEM micrograph showing four graphitic side walls. (c) Raman spectra (3 mW, $633 \mathrm{~nm}$ ) of the as-grown MWCNTs. No distinguishable RBM peaks were observed (100-500 $\mathrm{cm}^{-1}$ wave numbers not shown), relative to the spectrometers noise floor, suggesting a largely multiwall population.

that offer strong surface and intertube adhesion combined with excellent conductivities and nanotube alignment are advantageous traits. We herein report on the dry-transfer of multiwalled carbon nanotubes to polymer substrates and demonstrate control over the sheet resistance and optical transparency of the transferred thin films. Simple supercapacitor structures have also been demonstrated as one viable application of the proposed technique.

\section{Experiment}

Multiwalled carbon nanotubes (MWCNTs) were synthesised by thermal chemical vapour deposition (CVD) in a commercially available cold-walled reactor (AIXTRON, Black Magic). Bilayer catalysts were prepared by RF sputtering $10 \mathrm{~nm} \mathrm{Al}{ }_{2} \mathrm{O}_{x}$ onto $200 \mathrm{~nm}$ thermally oxidised Si substrates which were subsequently exposed to ambient atmosphere and coated with $1 \mathrm{~nm}$ thermally evaporated $\mathrm{Fe}(0.2 \AA / \mathrm{s})$. The chamber was prepressurised to $26 \mathrm{mbar}$ with $8 \mathrm{sccm}$ $\mathrm{C}_{2} \mathrm{H}_{2}$ diluted in $192 \mathrm{sccm} \mathrm{H} \mathrm{H}_{2}$. An ohmically heated graphite stage was ramped at $5^{\circ} \mathrm{C} / \mathrm{s}$ to $700^{\circ} \mathrm{C}$. Growth was repeatedly observed to initiate at $\sim 520^{\circ} \mathrm{C}$. Figures 1 (a) and 1(b) show typical profile and areal scanning electron micrographs (SEMs) of an as-grown MWCNT forest. A high-resolution transmission electron micrograph (HR-TEM) of an individual MWCNT is given in the inset of Figure 1(b). The MWCNTs are formed from $2-5$ graphitic walls, were $25 \pm 13$ (S.D) $\mathrm{nm}$ in diameter, and had an initial growth rate of $\sim 0.8 \mu \mathrm{m} / \mathrm{s}$ that monotonically decreased over time. Figure $1(\mathrm{c})$ is the Raman spectra of the as-grown forest (Renishaw InVia, $\mathrm{He}-\mathrm{Ne}$ source operating at $633 \mathrm{~nm} / 3 \mathrm{~mW}$ ). The spectra are characteristic of MWCNTs [22, 23] and were highly uniform across samples, highlighting process reproducibility.

Figure 2 illustrates the dry-transfer process. A polycarbonate (PC) destination substrate is angled toward the sources MWCNTs and is compressed using a quartz cylinder, rotated and sheared at $\sim 1 \mathrm{~cm} / \mathrm{s}$. The MWCNTs readily debond from the source substrate and adhere to the PC. The dry-transfer process does not degrade the crystallographic order of the MWCNTs, as evidenced via Raman spectroscopy. A variety of destination substrates were considered, including poly(ethylene terephthalate), polycarbonate, and $\mathrm{Al}$ foil. In the latter case, when transferring extremely short nanotubes $(<1 \mu \mathrm{m})$, self-assembled monolayer (SAM) adhesion promoters were necessary, such as poly(lysine) and aminopropyltriethoxy silane (APTES). Prior to SAM treatment MWCNTs did not adhere to the Al foil, whereas after treatment nearly $100 \%$ (by area) were successfully transferred.

Figure 3(c) shows a $900 \mathrm{~nm} \times 900 \mathrm{~nm}$ AFM micrograph (Agilent) of a dry-transferred MWCNT film on a PC substrate demonstrating the high degree of alignment. An optical micrograph of poly(lysine)-treated $\mathrm{Al}$ foil with a drytransferred MWCNT film is given in Figure 3(d). The inset depicts a schematic of the substrates cross-section.

MWCNT thin films were patterned by standard photolithography and $\mathrm{O}_{2}$ reactive ion etching (RIE: $0.2 \mathrm{mbar}$, $100 \mathrm{~W}, 120 \mathrm{~s}$ ) (Figures 3(b) and 3(e)). Transparency control was demonstrated using RIE. The transparency between the polymer-protected 5,20 , and $50 \mu \mathrm{m}$ dots was controlled, as illustrated in Figure 3(f). Transparency control was also demonstrated by adjusting the length of the MWCNTs prior to the dry-transfer, (by varying the CVD growth duration). In this instance, films were defined by patterning the bilayer catalyst prior to growth. A Mo catalyst-passivation process was used to prevent nanotube growth from particular areas. No nanotubes grew from those areas coated with Mo $(20 \mathrm{~nm})$. An inexpensive laser-jet patterning technique was employed to achieve this. Patterned MWCNT forests were then transferred, as afore mentioned. Repeatedly rolled films became sufficiently compacted such that they formed free-standing, pristine MWCNT "paper" that maintained structural integrity even under rigorous mechanical strain. 
(a)

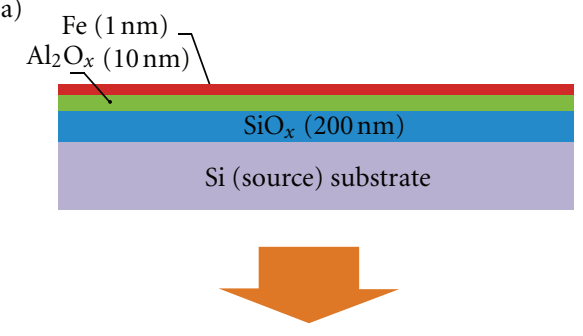

(b)

Vertical CNT forest

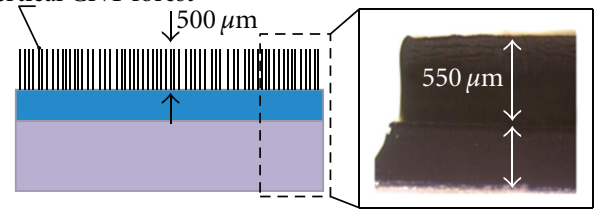

(c)
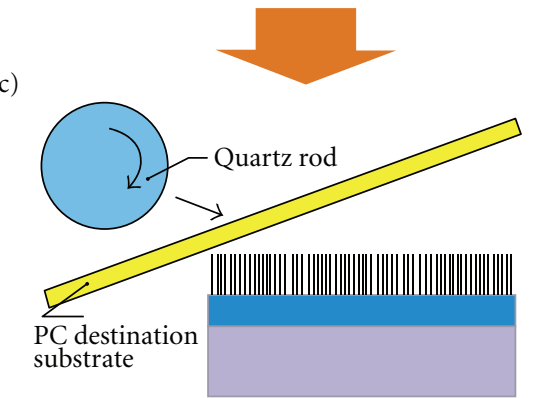

(d)

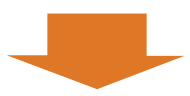

Aligned H-CNT film

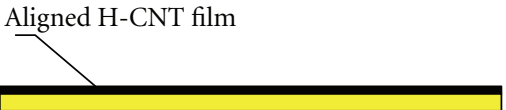

Figure 2: The dry-transfer process. (a) Cross-sectional illustration of the $\mathrm{Si} / \mathrm{SiO}_{2}(200 \mathrm{~nm})$ growth substrate magnetron sputtered with $10 \mathrm{~nm} \mathrm{Al}_{2} \mathrm{O}_{x} / 1 \mathrm{~nm}$ Fe thermally evaporated (Lesker PVD). (b) MWCNT synthesis by thermal CVD: $700^{\circ} \mathrm{C}, 26 \mathrm{mbar}, 192 \mathrm{sccm}$ $\mathrm{H}_{2}: 8 \mathrm{sccm} \mathrm{C}_{2} \mathrm{H}_{2}, 10 \mathrm{~min}$. Inset: Optical micrograph of an as-grown $550 \mu \mathrm{m}$ tall MWCNT forest. (c) PET destination substrate angled towards the source nanotube forest. PET and source nanotube substrate are compressed by a rolled quartz cylinder, moving at a rate of $\sim 1 \mathrm{~cm} / \mathrm{s}$. (d) Aligned MWCNT thin film.

Uniaxial strain and bend radii measurements were performed using computer-controlled custom-built systems connected to a Keithley 6430 source-measure unit. Experiments were performed at room temperature and pressure.

\section{Results and Discussions}

Figure 4 shows optical micrographs of three prepatterned transferred samples of differing MWCNT length. The sheet resistance $\left(R_{s}\right)$ and optical transmittance $(T)$ were found to be strongly dependent on the MWCNT length. Figure 5(a) shows the UV-Vis-NIR transmittance of films fabricated from MWCNTs $<1 \mu \mathrm{m}$ to $500 \mu \mathrm{m}$ in length. For the most opaque and highly conductive films, $R_{s} \sim 1.2 \Omega /$ sq. with $\sim 9 \%$ transparency $(550 \mathrm{~nm})$. For nominally equivalent samples, two- and four-probe conductivity measurements yielded
$R_{s} \sim 10.2 \Omega /$ sq. and $\sim 2.6 \Omega /$ sq., respectively, evidencing a low contact resistance $(7.6 \Omega / \mathrm{sq})$. The most transparent films ( $85 \%$ for $<1 \mu \mathrm{m}$ long MWCNTs) had sheet resistances as low as $550-1000 \Omega /$ sq. Reducing the nanotube length substantially increased the optical transmission whilst only increasing the sheet resistance by approximately one order of magnitude (Figure 5(b)). This observation can be explained in terms of the increased number of inter-nanotube junctions for films comprised of shorter nanotubes. Furthermore, one would expect longer nanotubes to increase the films conductivity as the rolling process produces substantially thicker films which offer lower bulk resistance. The DC conductivity in such films is primarily dependent on tunnelling between individual nanotubes. As a result, the conductivity is critically dependent on the number of conduction pathways, junctions, and the general network morphology-all of which can be varied by adjusting the nanotube length and degree of alignment. The films demonstrated optoelectronic characteristics nearing the ITO benchmark and superior characteristics to competing organic technologies such as TDA and PEG-enhanced PEDOT:PSS [24, 25].

The sheet resistance $\left(R_{s}\right)$ and optical transparency at $550 \mathrm{~nm}(T)$ are related by [26-28];

$$
T=\left[1+\left(\frac{t Z_{0}}{2}\right) \sigma_{\mathrm{opt}}\right]^{-2}=\left[1+188.5 \frac{1}{R_{s}}\left(\frac{\sigma_{\mathrm{opt}}}{\sigma_{\mathrm{dc}}}\right)\right]^{-2}
$$

where $Z_{0}$ is the impedance of free space $(377 \Omega), \sigma_{\mathrm{opt}}$ is the optical conductivity, and $\sigma_{\mathrm{dc}}$ is the direct current electrical conductivity. A viable transparent electrode must demonstrate low- $R_{s}$ and high- $T$ throughout the optical window (300-900 nm). For an ideal transparent conductor, $\left(\sigma_{\mathrm{opt}} / \sigma_{\mathrm{dc}}\right) \rightarrow 0$. Fitting $(1)$ gives $\left(\sigma_{\mathrm{opt}} / \sigma_{\mathrm{dc}}\right)=1.7 \times$ $10^{-1}$. Graphite has a conductivity ratio of $9.1 \times 10^{-2}$, whereas current driven applications require $4.5 \times 10^{-3}$. The minimum industry standard for (brittle) transparent conductors, typical for display applications, is $2.9 \times 10^{-2}$ ( $>90 \%$ for $<100 \Omega /$ sq.) $[26,29]$. The reported conductance ratio is someway off the industry standard but is nevertheless close to the state-of-the-art for thin films prepared from chemically modified graphene [26]. Though the pristine thin films offer only modest performance, it is important to stress that these films are chemically untreated. The films have not been doped in anyway. Suitable doping will lead to conductivity enhancements, for a given transparency, thus allowing the films to compete with more traditional vacuum and spray processing, which intrinsically employ en route doping procedures.

The AC sheet resistance of the dry-transferred films was investigated at microwave frequencies using a technique described in detail in [30]. The transmissive sheet resistances, in the $120-175 \mathrm{THz}$ range, were found to be 0.3 and $640 \Omega$ /sq., for nanotubes $500 \mu \mathrm{m}$ and $1 \mu \mathrm{m}$ long, and were broadly consistent with the DC measurements for films of similar optical characteristics discussed previously.

The uniaxial strain response of the dry-transferred MWCNTs and the primary competing technologies, namely CVD grown (2-4 layer) hot press laminated graphene (HPLG) [31] and commercially available PET-supported 

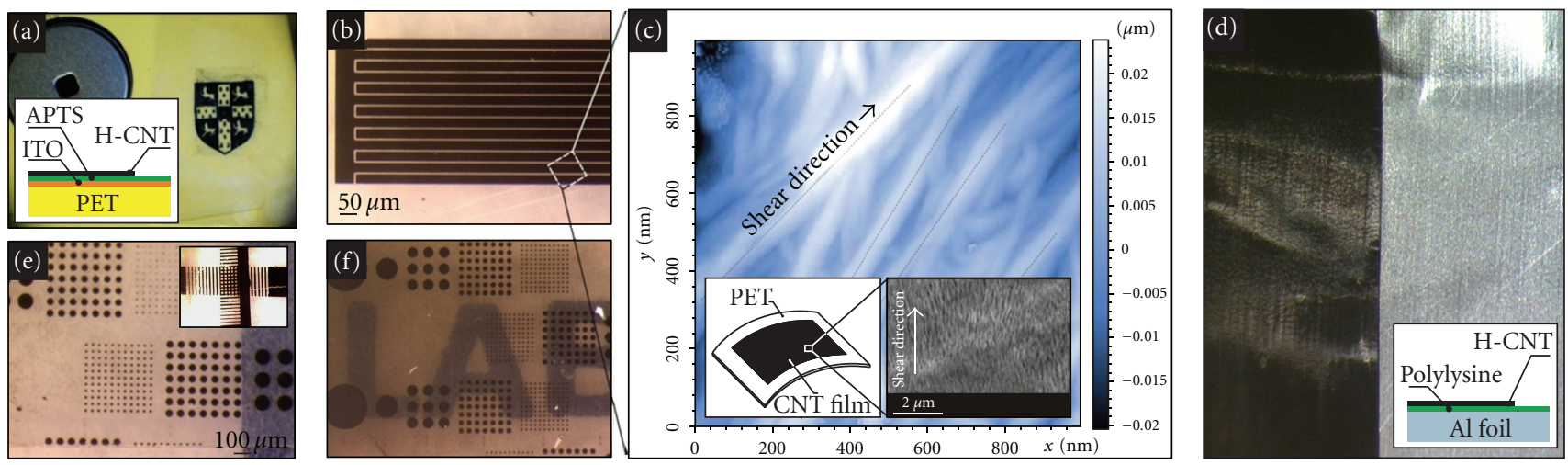

FIGURE 3: Patterned dry-transferred MWCNT thin films. (a) Patterning by Mo catalyst passivation. $\mathrm{Al}_{2} \mathrm{O}_{x} /$ Fe bilayer catalyst patterned using laser-jet/paper transfer and Mo deposition. Patterned MWCNT films were transferred to the destination PC (as described in Figure 2). A self-assembled monolayer, APTES was employed for PET/ITO substrates prior to transfer. (b, e) An aligned MWCNT film patterned into 5 $\mu \mathrm{m}$ interdigitated electrodes by standard photolithography and $\mathrm{O}_{2} \mathrm{RIE}(0.2 \mathrm{mbar}, 100 \mathrm{~W}, 120 \mathrm{~s})$. Inset: optical micrograph of a Mo-passivated prepatterned CNT film shear transferred to a PET substrate and a second perpendicularly aligned prepatterned forest shear transferred. (c) AFM micrograph $(900 \mathrm{~nm} \times 900 \mathrm{~nm})$ of a dry-transferred MWCNT film on a PC substrate. Inset: SEM micrograph of a transferred film showing the high level of macroscopic alignment. (d) Optical micrograph of poly(lysine)-treated (right) with a dry-transferred MWCNT film (left). Prior to treatment MWCNTs did not adhere. Inset: schematic of the substrate cross-section. (f) Transparency control by $\mathrm{O}_{2} \mathrm{RIE}$ with 5,20 , and $50 \mu \mathrm{m}$ dots defined by conventional photolithography.

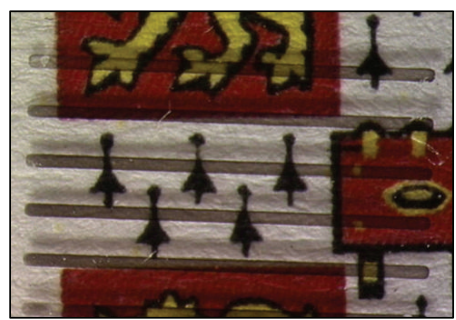

(a)

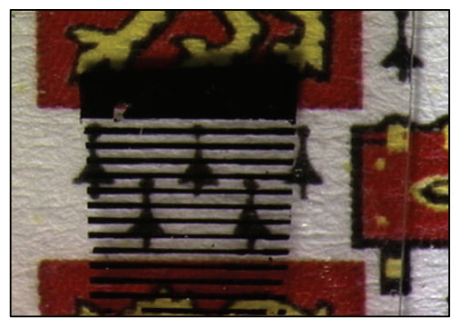

(b)

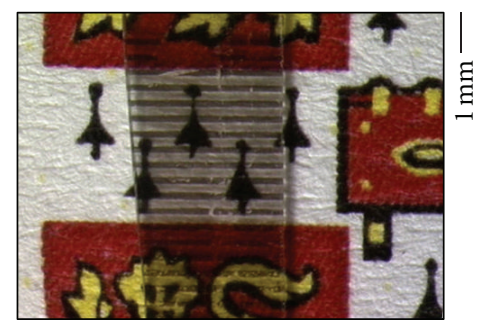

(c)

Figure 4: (a-c) Optical micrographs of Mo-passivated patterned MWCNT thin films transferred to PET showing controllable transparency by adjusting the length of the source nanotubes. The presented micrographs are for nanotubes that are (a, c) $30 \mu \mathrm{m}$ and (b) $500 \mu \mathrm{m}$ in length.
ITO, is shown in Figure 6(a). Samples were electrically contacted using mechanical clamps and conductive epoxy (inset Figure 6(a)). The maximum uniaxial tensile strain $(\varepsilon)$, defined as the strain for which $\partial(\Delta R / R) / \partial \varepsilon \rightarrow \infty$, of commercially available ITO was $\sim 1.2 \%$. HPLG samples failed at $\sim 15 \%$ and showed excellent correspondence with the theoretical values for single graphene flake failure [32, 33]. The dry-transferred MWCNT thin films withstood strains of up to $140 \%$ (the limit of the apparatus) whilst showing only a minor reduction in conductivity $\left(\Delta R / R_{o} \sim\right.$ 5 , where $R_{o}$ denotes the initial/relaxed sheet resistance). Similar performance has been reported elsewhere, though predominately only in the case of solution-processed singlewalled carbon nanotube thin films [34]. As the films become increasingly strained, the MWCNTs align. The individual MWCNTs observe negligible strain during this aligning stage. It is not until the film has maximised its degree of alignment that the MWCNTs undergo nanoscopic straining and eventually fail catastrophically. As a result, exceptionally high failure strains are possible owing to the networks porosity and anisotropy.

The cyclic strain performance is plotted in Figure 6(b). Under equivalent conditions, ITO/PET was strained to $1 \%$ and then relaxed. The resistivity of the ITO/PET was around $44 \%$ larger than the initial unstrained film. Straining irreversibly increased the sheet resistance of the ITO through microcrack formation. In contrast, dry-transferred films were strained (50\%), then relaxed, and the process repeated. The initial strain (cycle 1) increased the sample resistance by approximately $42 \%$; however each cycle thereafter the relaxed resistance repeatedly returned to this value. The longterm flexing stability of the dry-transferred thin films was considered by straining samples by $22 \%$, over 100 cycles. The dry-transferred films showed a negligible increase in 


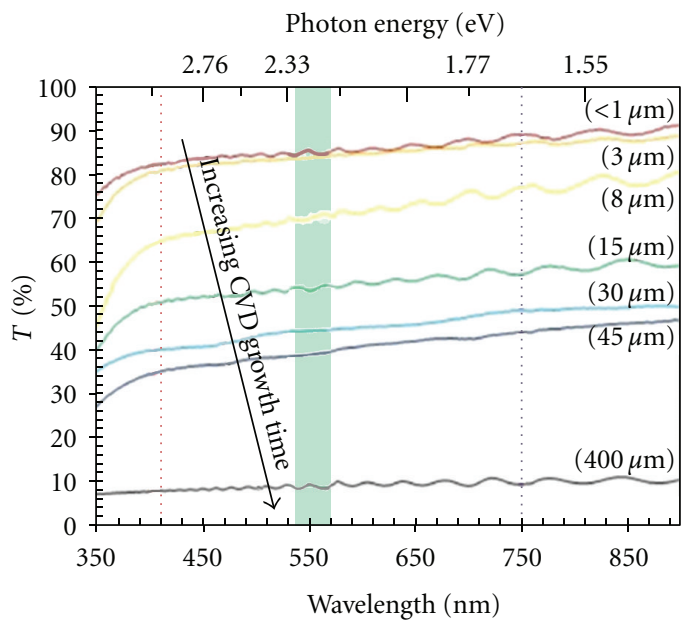

(a)

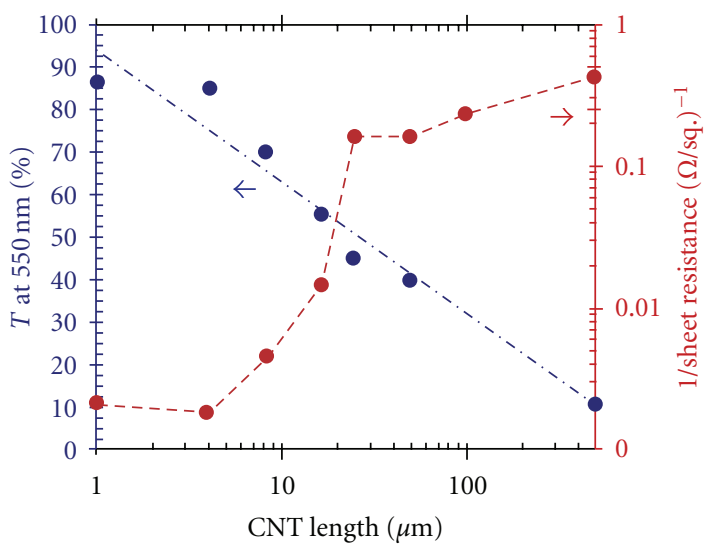

(b)

FIGURE 5: Opto-electronic performance. (a) UV-Vis-NIR. Variation in the optical transmission of dry-transfer MWCNT films consisting of MWCNTs of increasing source length. (b) Sheet resistance and optical transparency as a function of nanotube length.

resistance over time. Figure 6(c) depicts a dependence on the electrode separation. Increasing the electrode separation $(L)$ tended to increase the maximum attainable strain as this increased the amount of available material over which the strain was distributed. Figure 6(d) shows the variation in the normalised change in resistance $\left(\Delta R / R_{o}\right)$ as a function of the radius of curvature. The inset(s) depict the apparatus during a typical measurement. $\Delta R / R_{o}=0.09$ and was largely independent of the radius of curvature over the range considered. $\Delta R / R_{o}$ of the ITO samples increased by over three orders of magnitude for curvatures $<9.5 \mathrm{~mm}$. Similarly, HPLG samples showed a linear increase in $\Delta R / R_{o}$ for radius $<5 \mathrm{~mm}$.

A key feature of the reported technique is the ability to align the nanotubes during the inexpensive and rapid dry-transfer process. The degree of nanotube alignment is illustrated in Figure 7, which plots the Steridian 37 (Str37) function for a transferred sample, calculated using the SPIP image analysis software. Str37 is defined as the ratio of the in-plane distance between the real autocorrelation centre

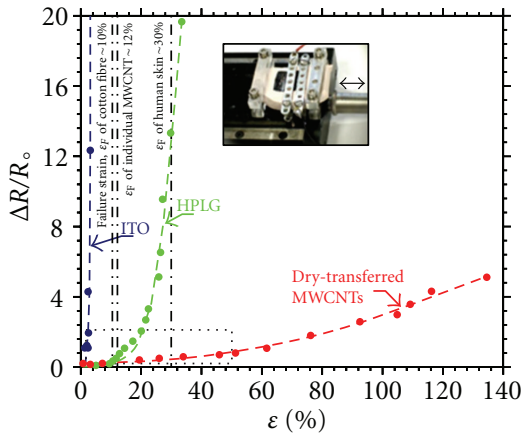

(a)

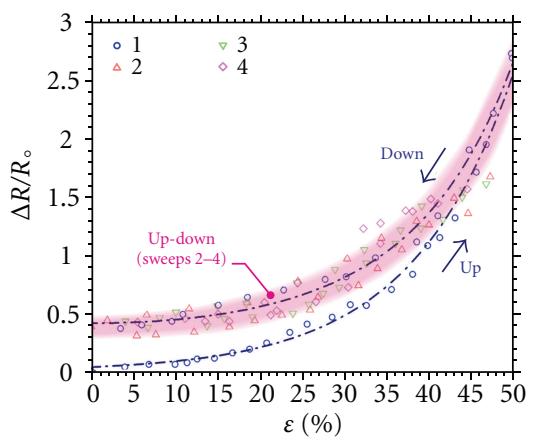

(b)

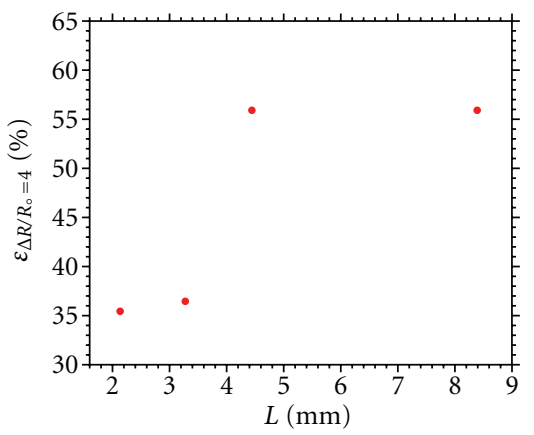

(c)

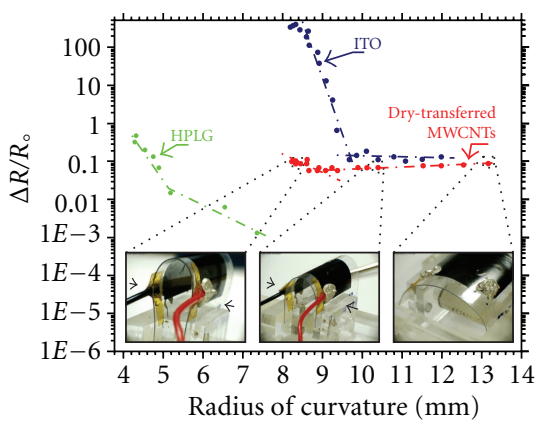

(d)

Figure 6: (a) Normalised resistance $\left(\Delta R / R_{o}\right)$ for ITO, hot press laminated grapheme (HPLG), and dry-transferred MWCNTs $(\sim 220 \mu \mathrm{m}$ in length) as a function of uniaxial strain. Failure strains of human skin, cotton fibre, and individual MWCNTs are included for comparison. (b) cyclic performance of a dry-transferred MWCNT thin film (for a fixed nanotube length of $130 \mu \mathrm{m}$ ). (c) Strain (at $\Delta R / R_{o}=4$ ) as a function of electrode separation. (d) Variation in sample resistance as a function of the radius of curvature. Insets: optical micrographs taken during a typical measurement. Note the lack of film de-binding, even under high curvature. 


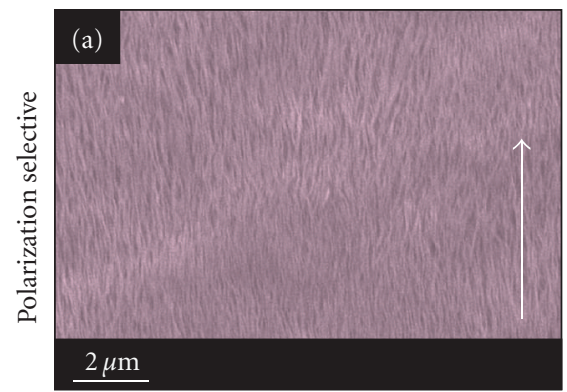

(b) $\operatorname{Str} 37=0.22$

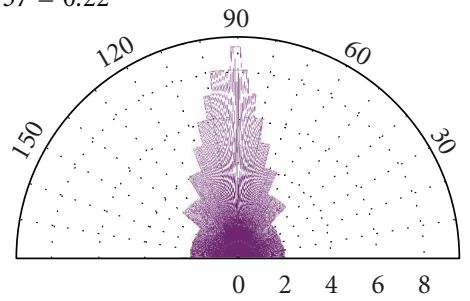

Correlation length (nm)

(d) $\operatorname{Str} 37=0.29$

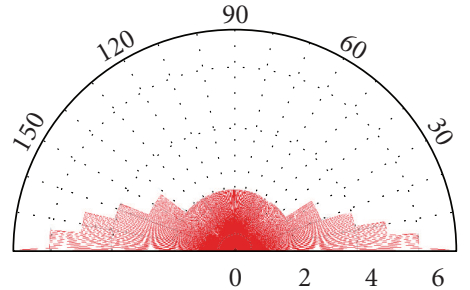

Correlation length (nm)

(f) $\operatorname{Str} 37=0.6$

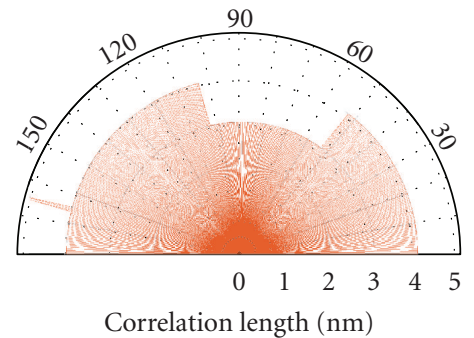

Correlation length (nm)

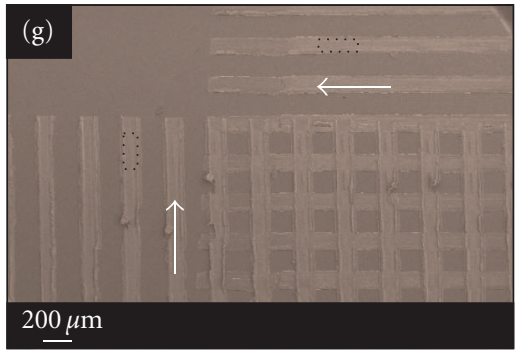

$200 \mu \mathrm{m}$
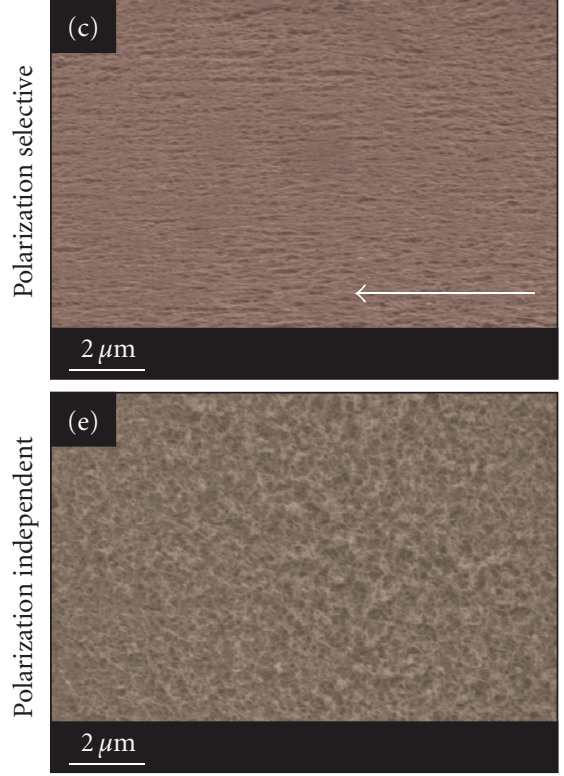

FIGURE 7: (a, c) SEM micrographs of the polarisation selective, partially transparent electrodes. (b, d) Str37 quantifying the alignment of the vertical (0.22) and horizontal (0.29) transfers, compared to (e, f) an unaligned compressed film (0.60). (g) SEM micrograph of crossedtransfers on a single untreated PET substrate. Arrows denote the transfer direction.

and the boundary at which the height of the image has decayed to $37 \%$ of the depth-range at this autocorrelation centre. An autocorrelation is applied to the images depthrange to infer the position. The decay in the depth-range in all directions is evaluated and the Str37 value is calculated $[35,36]$. For perfectly aligned arrays Str37 $=0$, whilst for disordered networks which exhibit no directional preference, Str37 $=1$. Depending on the roll technique both wellaligned (Figures $7(\mathrm{a})-7(\mathrm{~d})$ ) and highly isotropic networks could be transferred (Figures $7(\mathrm{e})$ and $7(\mathrm{f})$ ).. tr $37=0.26$ for the aligned transfers and 0.60 for the compressed/isotropic transfers.

Aligned nanotube electrodes may find application as tunable optical polorisors and advanced electrode materials in next generation flexible display technologies. Figure 7(g) shows a fabricated bilayer structure. Here crossed and aligned structures, of controlled controlled pitch, were transferred to a PET substrate by successive rolls of pre-patterned forests to from optical polarisers that exploit the nanoscopic spacing of the nanotubes, and also the microscopic spacing of the patterned films.

To demonstrate one potential application of the drytransferred thin films, two-electrode electric double-layer
(EDLC) cells were constructed. Due to the films' high conductivity, the MWCNTs functioned as both the active material and charge collector, reducing the overall material requirements therefore increasing the weight fraction of the active material in the device. The final EDLC was $10 \mathrm{~mm} \times 10 \mathrm{~mm}$ (Figure 8(a)). An insulating porous separator (Celgard 2500) was sandwiched between two MWCNT electrodes soaked in electrolyte $\left(1 \mathrm{M}\right.$ Tetraethylammonium tetrafluoroborate $\left(\mathrm{Et}_{4} \mathrm{NBF}_{4}\right)$ in propylene carbonate, Sigma-Aldrich). Al tape was used to externally electrically contact the cell. Three different films, comprised of different MWCNT lengths, were considered. The nanotube lengths and active weights were $28.5 \mu \mathrm{m} / 0.6 \mathrm{mg} \cdot \mathrm{cm}^{-2}$ (1 min growth), $96 \mu \mathrm{m} / 1.1 \mathrm{mg} \cdot \mathrm{cm}^{-2}$ (5 min), and $235 \mu \mathrm{m} / 2.3 \mathrm{mg} \cdot \mathrm{cm}^{-2}$ (10 min). Cells were charcaterised by galvanostatic charge/discharge $\left(1 \mathrm{~mA} / \mathrm{cm}^{2}\right)$ and cyclic voltammetry $(50 \mathrm{mV} / \mathrm{s})$ measured using an Autolab PGSTAT302N potentiostat (Figure 8(b)).

Thicker films offered higher conductivities and longer nanotubes showed a higher capacitance per unit area as well as a lower equivalent series resistance (ESR), both of which are essential for high-power delivery. The capacitance of the two-electrode MWCNT EDLCs was estimated to be between 


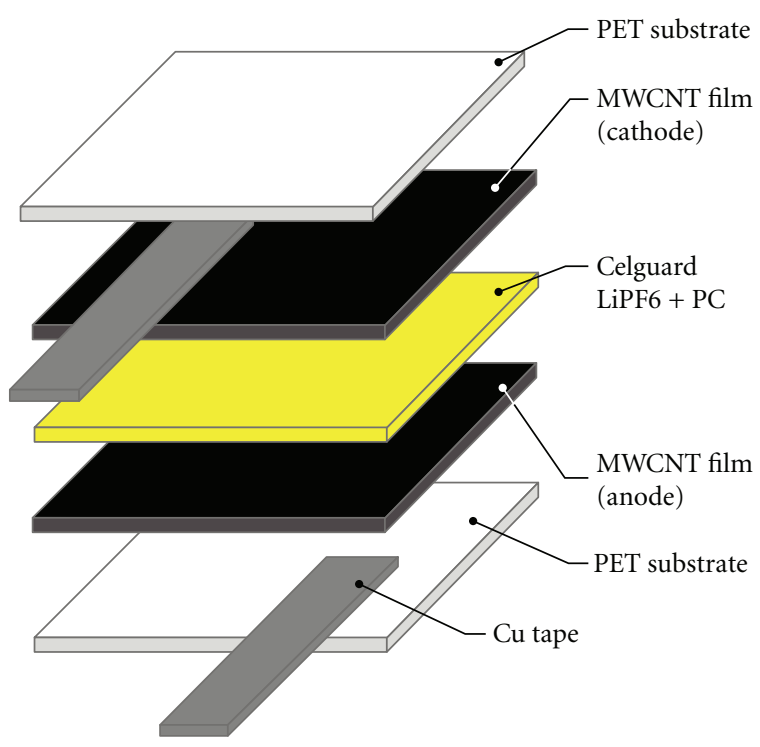

(a)

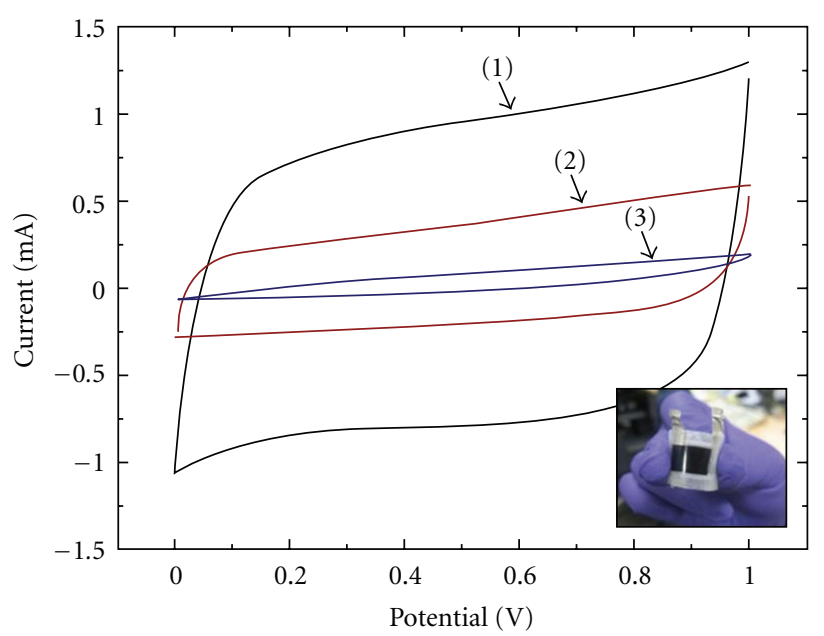

- (1) $10 \mathrm{~min}$
- (2) $5 \mathrm{~min}$
- (3) $1 \mathrm{~min}$

(b)

FIGURE 8: Dry-transferred MWCNT supercapacitor. (a) Schematic of the supercapacitor structure using a Celgard 2500 porous separator soaked in $\mathrm{Et}_{4} \mathrm{NBF}_{4}$. (b) Cyclic voltammetry for aligned MWCNT electrodes synthesised for (1) $10 \mathrm{~min}$, (2) $5 \mathrm{~min}$ and (3) $1 \mathrm{~min}$. The inset shows a fabricated device under flexing.

6 and $17 \mathrm{~F} / \mathrm{g}$, for cells biased to $1 \mathrm{~V}$. This is roughly twice the capacitance reported for sprayed, solution-processed singlewalled carbon nanotube devices under similar experimental conditions [37]. The ESR decreases with increasing film conductivity and ranged from $1000 \Omega(28.5 \mu \mathrm{m})$ to $44 \Omega$ $(235 \mu \mathrm{m})$ corroborating the sheet resistance estimates made previously. Nevertheless, the overall ESR is still somewhat higher than expected. This is most likely attributed to the relatively resistive Schottky barrier at the Al-MWCNT interface, the intrinsic resistivity of the electrolyte, as well as contributions from the multiple electrolyte/MWCNT interfaces. Thicker electrodes increase the energy density allowing the active material to occupy a greater volume ratio. However, this is offset by higher ion diffusion barriers in the inner regions of the electrode, resulting in substantial internal resistance and consequently reducing high-power performance. This limits the practical thickness of any nanostructured electrode. Thus, ideally thick electrodes that possess both high capacitance and that are operable at high power are desired. Up to the thicknesses studied, approximately constant power densities were observed $(2.48 \mathrm{~W} / \mathrm{g})$, indicating that high permeability is maintained even for thicker electrodes.

\section{Conclusions}

We have reported the development of an MWCNT drytransfer technique capable of transferring CVD-synthesised aligned MWCNTs to a variety of flexible substrates without chemically pre-treating the MWCNTs to enhance their adhesion. Accurate control over both the optical transparency and sheet resistance was shown to depend critically on the length of the as-grown MWCNTs. The dry-transferred MWCNT thin films showed a change in resistance as low as 5 for strains in excess of $140 \%$, outperforming PET-supported ITO by two orders of magnitude and hot press laminated graphene by one order of magnitude. The alignment of the constituent MWCNTs was shown to be controllable and was demonstrated to offer a viable approach to the fabrication of flexible transparent polarisation selective electrodes for advanced display technologies. Double-layer supercapacitor cells were fabricated and showed a modest capacitance of up to $17 \mathrm{~F} / \mathrm{g}$. The results demonstrated that the dry-transferred MWCNT electrodes are excellent candidates for inexpensive, compact, high-energy-density storage and offer industry compatibility via a facile active material transfer approach.

\section{Acknowledgments}

This work was supported by the Schiff Studentship, Cambridge University. M. Cole acknowledges the support of St. John's College Cambridge and St. Edmund's College Cambridge. C. Li thanks the Scientific Research Foundation of Graduate School of Southeast University, China (Grant no. YBJJ0926). K. Teo acknowledges the support of the EC Grant TECHNOTUBE.

\section{References}

[1] D. Zhang, K. Ryu, X. Liu et al., "Transparent, conductive, and flexible carbon nanotube films and their application in organic light-emitting diodes," Nano Letters, vol. 6, no. 9, pp. 18801886, 2006.

[2] K. Bradley, J. C. P. Gabriel, and G. Grüner, "Flexible nanotube electronics," Nano Letters, vol. 3, no. 10, pp. 1353-1355, 2003.

[3] C. Qing, K. Hoon-sik, N. Pimparkar et al., "Medium-scale carbon nanotube thin-film integrated circuits on flexible plastic substrates," Nature, vol. 454, no. 7203, pp. 495-500, 2008. 
[4] W. Zhuangchun, C. Zhihong, X. Du et al., "Transparent, conductive carbon nanotube films," Science, vol. 305, no. 5688, pp. 1273-1276, 2004.

[5] Y. Zhou, L. Hu, and G. Grüner, "A method of printing carbon nanotube thin films," Applied Physics Letters, vol. 88, no. 12, Article ID 123109, 2006.

[6] L. Hu, D. S. Hecht, and G. Gruner, "Percolation in transparent and conducting carbon nanotube networks," Nano Letters, vol. 4, no. 12, pp. 2513-2517, 2004.

[7] E. Artukovic, M. Kaempgen, D. S. Hecht, S. Roth, and G. Grüner, "Transparent and flexible carbon nanotube transistors," Nano Letters, vol. 5, no. 4, pp. 757-760, 2005.

[8] H. E. Unalan, G. Fanchini, A. Kanwal, A. D. Pasquier, and M. Chhowalla, "Design criteria for transparent single-wall carbon nanotube thin-film transistors," Nano Letters, vol. 6, no. 4, pp. 677-682, 2006.

[9] B. B. Parekh, G. Fanchini, G. Eda, and M. Chhowalla, "Improved conductivity of transparent single-wall carbon nanotube thin films via stable postdeposition functionalization," Applied Physics Letters, vol. 90, no. 12, Article ID 121913, 2007.

[10] S. Hofmann, C. Ducati, B. Kleinsorge, and J. Robertson, "Direct growth of aligned carbon nanotube field emitter arrays onto plastic substrates," Applied Physics Letters, vol. 83, no. 22, pp. 4661-4663, 2003.

[11] S. Hofmann, C. Ducati, J. Robertson, and B. Kleinsorge, "Lowtemperature growth of carbon nanotubes by plasma-enhanced chemical vapor deposition," Applied Physics Letters, vol. 83, no. 1, pp. 135-137, 2003.

[12] P. Beecher, P. Servati, A. Rozhin et al., "Ink-jet printing of carbon nanotube thin film transistors," Journal of Applied Physics, vol. 102, no. 4, Article ID 043710, 2007.

[13] N. P. Armitage, J. C. P. Gabriel, and G. Grüner, "Quasi-Langmuir-Blodgett thin film deposition of carbon nanotubes," Journal of Applied Physics, vol. 95, no. 6, pp. 3228-3230, 2004.

[14] M. A. Meitl, Y. Zhou, A. Gaur et al., "Solution casting and transfer printing single-walled carbon nanotube films," Nano Letters, vol. 4, no. 9, pp. 1643-1647, 2004.

[15] M. Chhowalla, K. B. K. Teo, C. Ducati et al., "Growth process conditions of vertically aligned carbon nanotubes using plasma enhanced chemical vapor deposition," Journal of Applied Physics, vol. 90, no. 10, pp. 5308-5317, 2001.

[16] Z. F. Ren and Z. P. Huang, "Synthesis of large arrays of wellaligned carbon nanotubes on glass," Science, vol. 282, no. 5391, pp. 1105-1107, 1998.

[17] C. J. Strobl, C. Schäflein, U. Beierlein, J. Ebbecke, and A. Wixforth, "Carbon nanotube alignment by surface acoustic waves," Applied Physics Letters, vol. 85, no. 8, pp. 1427-1429, 2004.

[18] S. Bhattacharyya, E. Kymakis, and G. A. J. Amaratunga, "Photovoltaic properties of dye functionalized single-wall carbon nanotube/conjugated polymer devices," Chemistry of Materials, vol. 16, no. 23, pp. 4819-4823, 2004.

[19] W. X. Chen, J. Y. Lee, and Z. Liu, "The nanocomposites of carbon nanotube with $\mathrm{Sb}$ and $\mathrm{SnSb}_{0.5}$ as Li-ion battery anodes," Carbon, vol. 41, no. 5, pp. 959-966, 2003.

[20] C. Du, J. Yeh, and N. Pan, "High power density supercapacitors using locally aligned carbon nanotube electrodes," Nanotechnology, vol. 16, no. 4, pp. 350-353, 2005.

[21] V. Gupta and N. Miura, "Polyaniline/single-wall carbon nanotube (PANI/SWCNT) composites for high performance supercapacitors," Electrochimica Acta, vol. 52, no. 4, pp. 17211726, 2006.
[22] A. M. Rao, A. Jorio, M. A. Pimenta et al., "Polarized Raman study of aligned multiwalled carbon nanotubes," Physical Review Letters, vol. 84, no. 8, pp. 1820-1823, 2000.

[23] E. F. Antunes, A. O. Lobo, E. J. Corat, and V. J. Trava-Airoldi, "Influence of diameter in the Raman spectra of aligned multiwalled carbon nanotubes," Carbon, vol. 45, no. 5, pp. 913-921, 2007.

[24] B. D. Martin, N. Nikolov, S. K. Pollack et al., "Hydroxylated secondary dopants for surface resistance enhancement in transparent poly(3,4-ethylenedioxythiophene)-poly(styrenesulfonate) thin films," Synthetic Metals, vol. 142, no. 1-3, pp. 187-193, 2004.

[25] F. C. Krebs and B. Winther-Jensen, "High-conductivity largearea semi-transparent electrodes for polymer photovoltaics by silk screen printing and vapour-phase deposition," Solar Energy Materials and Solar Cells, vol. 90, no. 2, pp. 123-132, 2006.

[26] S. De, P. J. King, M. Lotya et al., "Flexible, transparent, conducting films of randomly stacked graphene from surfactantstabilized, oxide-free graphene dispersions," Small, vol. 6, no. 3, pp. 458-464, 2010.

[27] S. De, T. M. Higgins, P. E. Lyons et al., "Silver nanowire networks as flexible, transparent, conducting films: extremely high DC to optical conductivity ratios," ACS Nano, vol. 3, no. 7, pp. 1767-1774, 2009.

[28] E. M. Doherty, S. De, P. E. Lyons et al., “The spatial uniformity and electromechanical stability of transparent, conductive films of single walled nanotubes," Carbon, vol. 47, no. 10, pp. 2466-2473, 2009.

[29] L. Gao, W. Ren, J. Zhao, L. P. Ma, Z. Chen, and H. M. Cheng, "Efficient growth of high-quality graphene films on $\mathrm{Cu}$ foils by ambient pressure chemical vapor deposition," Applied Physics Letters, vol. 97, no. 18, Article ID 183109, 3 pages, 2010.

[30] R. J. Collier and D. G. Hasko, "Measurement of the sheet resistance of resistive films on thin substrates from 120 to $175 \mathrm{GHz}$ using dielectric waveguides," Journal of Applied Physics, vol. 91, no. 4, p. 2547, 2002.

[31] S. Bae, H. Kim, Y. Lee et al., "Roll-to-roll production of 30inch graphene films for transparent electrodes," Nature Nanotechnology, vol. 5, no. 8, pp. 574-578, 2010.

[32] C. Lee, X. Wei, J. W. Kysar, and J. Hone, "Measurement of the elastic properties and intrinsic strength of monolayer graphene," Science, vol. 321, no. 5887, pp. 385-388, 2008.

[33] V. M. Pereira, A. H. Castro Neto, and N. M. R. Peres, “Tightbinding approach to uniaxial strain in graphene," Physical Review B, vol. 80, no. 4, Article ID 045401, 8 pages, 2009.

[34] L. Hu, W. Yuan, P. Brochu, G. Gruner, and Q. Pei, "Highly stretchable, conductive, and transparent nanotube thin films," Applied Physics Letters, vol. 94, no. 16, Article ID 161108, 3 pages, 2009.

[35] Q. Zhang, P. Vichchulada, and M. D. Lay, "Effect of deposition conditions on percolation in single-walled carbon nanotube networks," Physica Status Solidi A, vol. 207, no. 3, pp. 734-738, 2010.

[36] P. Vichchulada, Q. H. Zhang et al., "Macroscopic electrical properties of ordered single-walled carbon nanotube networks," Applied Materials Interfaces, vol. 2, pp. 467-473, 2010.

[37] M. Kaempgen, C. K. Chan, J. Ma, Y. Cui, and G. Gruner, "Printable thin film supercapacitors using single-walled carbon nanotubes," Nano Letters, vol. 9, no. 5, pp. 1872-1876, 2009. 

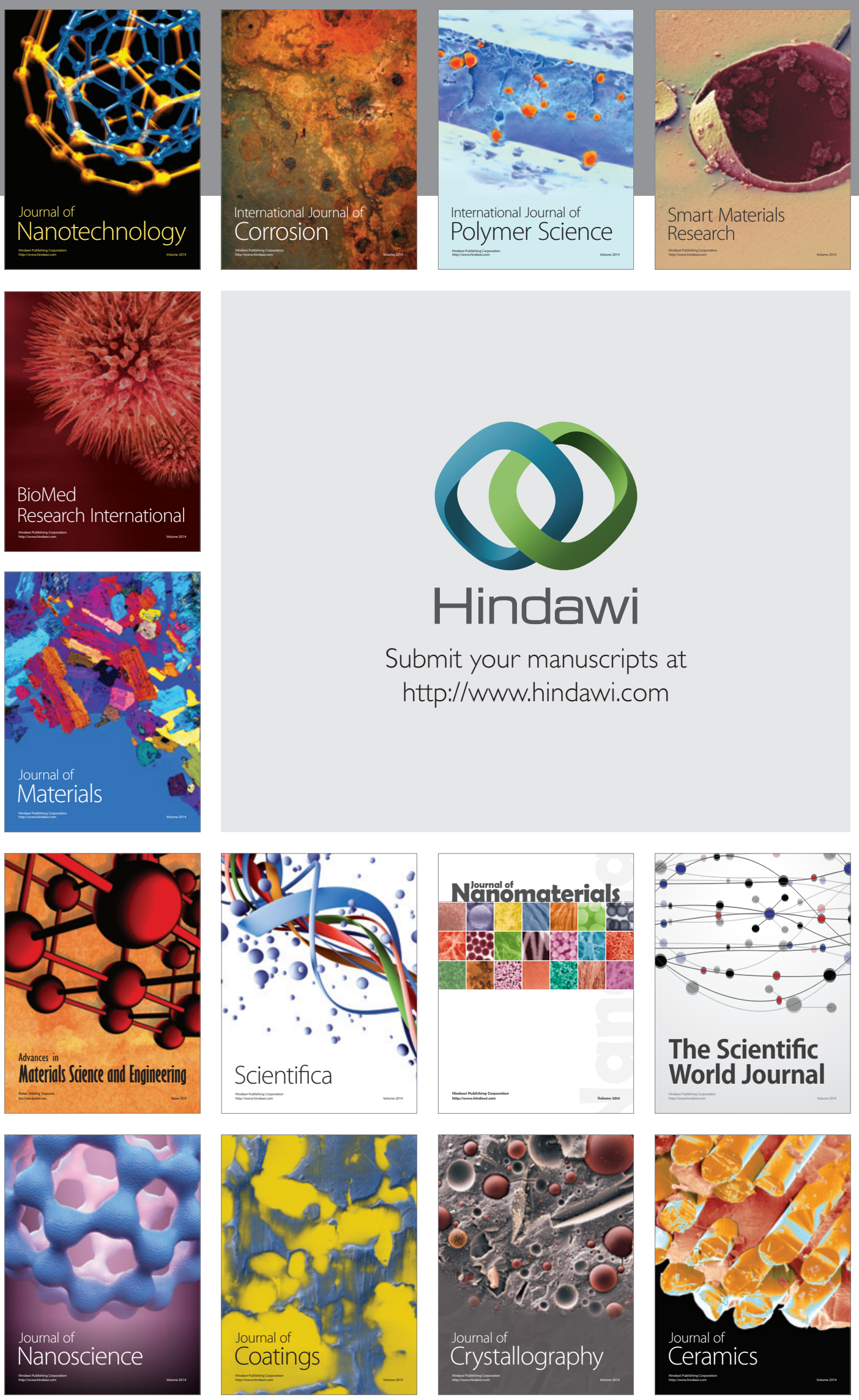

The Scientific World Journal

Submit your manuscripts at

http://www.hindawi.com

\section{World Journal}

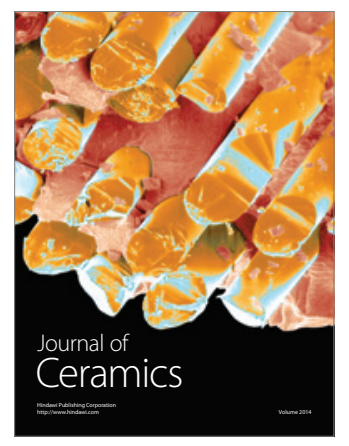

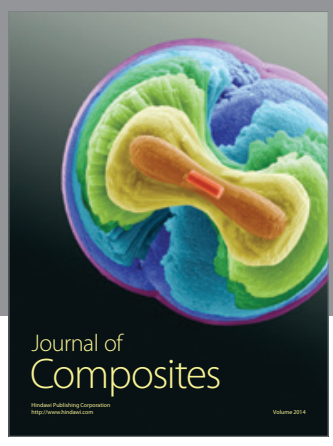
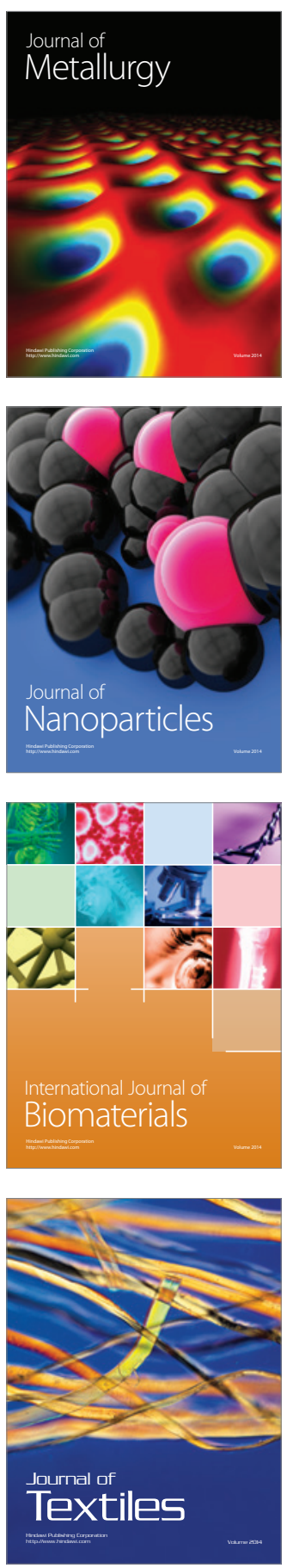